\title{
Fluid-Structure Interaction Analysis of Hydrofoils in a Pulsating Flow
}

\author{
Jiasheng $\mathrm{Li}^{1}$, Paul Croaker ${ }^{2}$, Jin Tian ${ }^{1}$, Mahmoud Karimi ${ }^{2}$ and Hongxing Hua ${ }^{1}$ \\ ${ }^{1}$ State Key Laboratory of Mechanical System and Vibration, Shanghai Jiao tong University, Shanghai 200240, PR China \\ ${ }^{2}$ School of Mechanical and Manufacturing Engineering, UNSW Australia, Sydney, Australia
}

\begin{abstract}
The reduction of noise and vibration are very important in the design of hydrofoils. The current study focuses on establishing a theoretical and numerical model to investigate fluid-structure interaction caused by elastic hydrofoils in a pulsating flow. A fully coupled three dimensional boun dary element method (BEM) and finite element method (FEM) code is applied to analyze the hydrodynamic performance. The numerical results show that the peak frequencies of the support reactions are related to the nat ural frequency of the hydrofoil. The natural frequencies and support reaction amplitudes are reduced significantly by including the fluid-structure coupling.
\end{abstract}

\section{Introduction}

\subsection{Previous work}

Fluid-structure interaction has become an important research area in the field of underwater structures and vehicles. Although determining the hydrodynamic loads acting on an elastic hydrofoil is still quite challenging, a number of experimental and numerical studies have been devoted to this topic. Jiho You et al. [1] and Suzanne [2] investigated the performance of elastic hydrofoil by CFD. Young [3, 4] and Lin et al. [5] used potential flow theory to predict the coupled flu id-structural response of elastic propellers under cavitating and non-cavitating conditions. Liu et al. [6] and Torre et al. [7] conducted an experimental study into the hydrodynamic characteristics of an oscillating hydrofoil.

\subsection{Objective of the present work}

The present work aims to investigate the fluid-structure interaction of a hydrofoil in a pulsating flow. The methodology is presented in Section 2, hydrodynamic performances are given in Section 3 and the main findings are summarised in Section 4.

\section{Mathematical model}

\subsection{Structural model}

A hydrofoil with one end free and the other end fixed is considered in this model. By using classical finite element theory, the discrete equation of motion for the hydrofoil can be written as:

$$
[M]\left|\ddot{\Delta}_{i}\right|+[C]\left|\dot{\Delta}_{i}\right|+[K]\left|\Delta_{i}\right|=\left|F_{w}\right|
$$
where $\left|\ddot{\Delta}_{i}\right|,\left|\dot{\Delta}_{i}\right|$ and $\left|\Delta_{i}\right|$ are the nodal acceleration, velocity, and displacement vectors, respectively. $[M]$, $[C]$ and $[K]$ are the global structure mass, damping, and stiffness matrices. $\left|F_{w}\right|$ is the hydrodynamic force vector generated by the pulsating flow.

\subsection{Pulsating flow}

The pulsating flow is generated by assigning the following time-dependent velocity

$$
\boldsymbol{V}(x, y, z, t)=\boldsymbol{V}_{0}+\boldsymbol{A}_{p}(x, y, z) \cos (\omega t)
$$

where, $V_{0}$ represents the uniform component of the inflow. $\boldsymbol{A}_{p}(x, y, z)$ and $\omega$ are respectively the amplitude and frequency of the pulsating flow. $x, y, z$ are spatial coordinates, and $t$ denotes time.

\subsection{Hydrodynamic model}

In order to calculate the hydrodynamic force $\left|F_{w}\right|$, a BEM method is used to solve the boundary-value problems given by:

$$
\begin{gathered}
\nabla^{2} \phi=0(\text { in } \Omega) \\
-\frac{\partial \phi}{\partial \boldsymbol{n}}=\boldsymbol{V} \cdot \boldsymbol{n}-\frac{\partial \boldsymbol{\delta}}{\partial t} \cdot \boldsymbol{n}(\text { on } \Gamma) \\
\Delta \phi_{w}\left(\boldsymbol{R}_{\text {wake }}, t\right)=\Delta \phi\left(\boldsymbol{R}_{r e}, t-t^{\prime}\right)(\text { Kutta condition })
\end{gathered}
$$

Here, $\phi$ is the hydrofoil-induced perturbation potential, $\boldsymbol{n}$ is the outward unit normal vector, $\boldsymbol{\delta}$ is the displacement vector of nodes on the hydrofoil 
surface. $\Delta \phi_{w}\left(\boldsymbol{R}_{\text {wake }}, t\right)$ represents the potential jump across the wake sheets, $\Delta \phi\left(\boldsymbol{R}_{r e}, t-t^{\prime}\right)$ is the potential jump across the hydrofoil surface at trailing edge. $t^{\prime}$ denotes the time required for the fluid to travel along the wake surface from the hydrofoil trailing edge $\boldsymbol{R}_{r e}$ to the wake point $\boldsymbol{R}_{\text {wake }}$.

The Laplace equation is combined with the MorinaKutta condition to determine the hydrofoil-induced perturbation potential, and kinematic boundary conditions under the small deformation hypothes is ( see more details in $[3,4,10])$.

\subsection{Hydrodynamic force}

The perturbation potential, $\phi$, obtained in the preceding section is applied to the linearized Bernoulli equation:

$$
p=-\rho\left(\frac{\partial \phi}{\partial t}+\boldsymbol{V}_{0} \cdot \nabla \phi\right)
$$

to predict the pressure fluctuation on the hydrofoil surface. These hydrodynamic forces are then decomposed into two parts :

$$
\left|F_{w}\right|=\left|F_{w}^{1}\right|+\left|F_{w}^{2}\right|
$$

Here, $\left|F_{w}^{1}\right|$ is a fuction of $\left|\ddot{\Delta}_{i}\right|,\left|\dot{\Delta}_{i}\right|$ and $\left|\Delta_{i}\right|$, and can be expanded as $-\left(\left[M_{w}\right]\left|\ddot{\Delta}_{i}\right|+\left[C_{w}\right]\left|\dot{\Delta}_{i}\right|+\left[K_{w}\right]\left|\Delta_{i}\right|\right)$ which describes the interaction between fluid and structure. $\left[M_{w}\right],\left[C_{w}\right]$ and $\left[K_{w}\right]$ are the added-mass, addeddamping and added-stiffness matrices. Meanwhile, $\left|F_{w}^{2}\right|$ has no relationship with the structure and provides excitation for the fluid-structure coupling system.

\subsection{Governing equation}

By moving $\left|F_{w}^{1}\right|$ to the left side of equation (1), the governing equation becomes

$$
\left([M]+\left[M_{w}\right]\right)\left|\ddot{\Delta}_{i}\right|+\left([C]+\left[C_{w}\right]\right)\left|\dot{\Delta}_{i}\right|+\left([K]+\left[K_{w}\right]\right)\left|\Delta_{i}\right|=\left|F_{w}^{2}\right|
$$

By Appling a FEM code co mbined with a BEM code, $[M],\left[M_{w}\right],[C],\left[C_{w}\right],[K],\left[K_{w}\right],\left|F_{w}^{2}\right|$ can be calculated, with the dynamic response predicted using Wilson-theta method in classical vibration theory.

\section{Results and discussions}

\subsection{Symmetric hydrofoil}

The combined BEM [8, 9, 11-13] and FEM method has been used to calculate a NACA 0015 hydrofoil (Fig. 1) moving in a pulsating flow with various frequencies given by $\omega=n \pi, n=2,4, \ldots, 28,30$. The hydrofoil has one end fixed (shown in blue in Fig. 1) and the other end free. The chord length is $1 \mathrm{~m}$ and its span length is $4 \mathrm{~m}$. The density, Poisson's ratio and elastic modulus of the hydrofoil are $7800 \mathrm{~kg} / \mathrm{m}^{3}, \quad 0.3$ and $2.1 \times 10^{11} \mathrm{~Pa}$, respectively. In this work, the damping matrix of the hydrofoil is neglected. The incoming uniform velocity is $10 \mathrm{~m} / \mathrm{s}$, angle of attack is $\alpha=10^{\circ}$, and amplitude of the pulsating flow is $0.1 \mathrm{~m} / \mathrm{s}$ in the $\mathrm{z}$ direction. The first natural frequency of the hydrofoil is $7.71 \mathrm{~Hz}$ without considering the fluid-structure coupling matrices and $5.59 \mathrm{~Hz}$ when those matrices are considered. As the support reactions in the $\mathrm{x}$ and $\mathrm{y}$ directions are very small, only the support reactions in the $\mathrm{z}$ direction are presented. Figure 2 shows the impact of the fluid-structure coupling matrices on the reaction force amplitudes as a function of pulsating flow frequency.

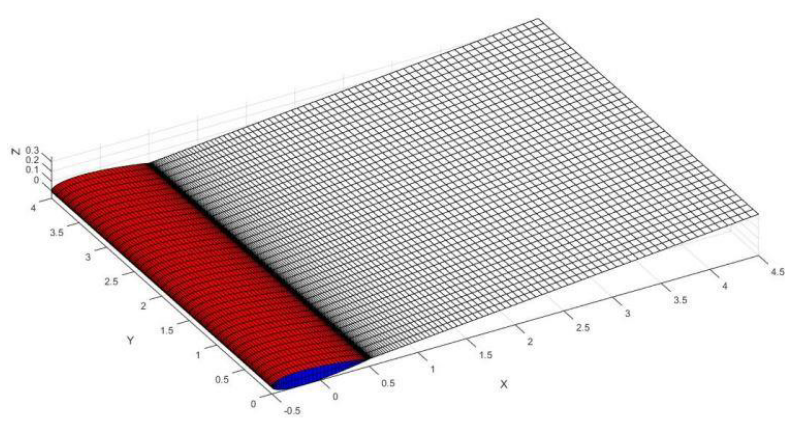

Figure 1. Discretization of symmetric NACA 0015 hydrofoil

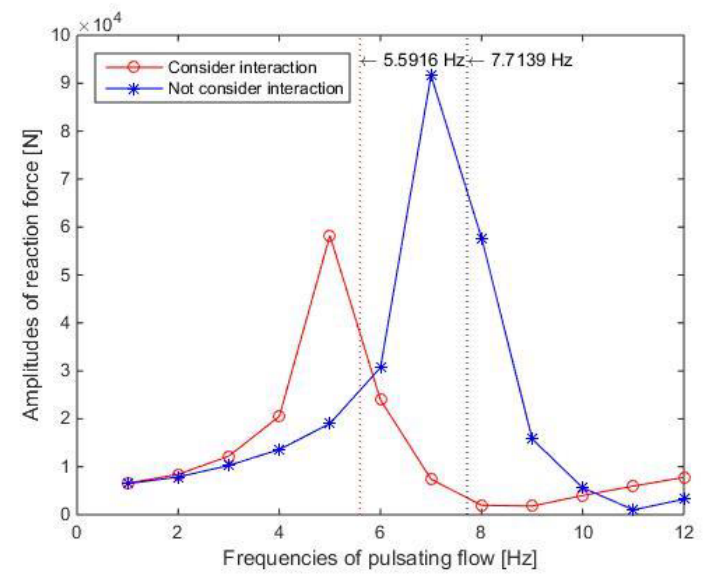

Figure 2. Reaction amplitudes for varying pulsating flow frequency for the symmetric NACA0015 hydrofoil.

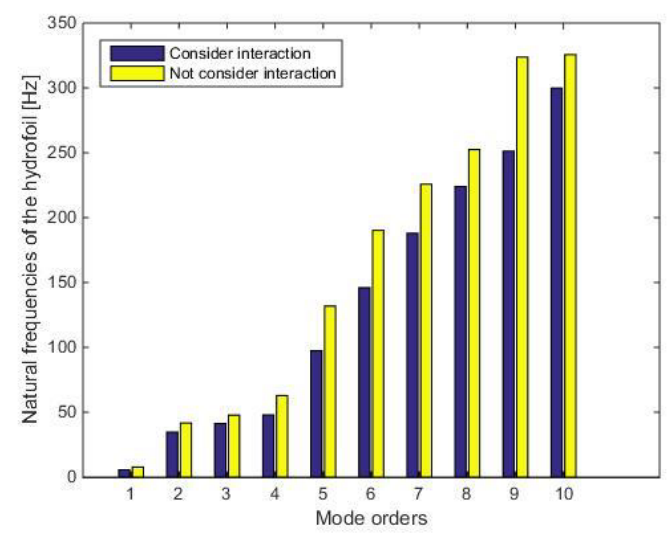

Figure 3. Natural frequency comparison for the symmetric NACA 0015 hydrofoil. 
Figure 2 shows that:

1. The maximum reaction force occurs when the pulsating flow frequency matches the first natural frequency of the hydrofoil. This is true regardless of whether the fluid-structure coupling matrices are included. There is no contradiction with classical vibration theory.

2. Including the fluid-structure coupling matrices reduces the first natural frequency by approximately $27.5 \%$. As Figure 3 shows, the other natural frequencies are also considerably reduced, by including the fluid-structure coupling matrices.

3. The amplitude of the support reaction at the first natural frequency is $36 \%$ less when the fluidstructure coupling matrices are considered.

\subsection{Asymmetric hydrofoil}

For comparison, the combined codes are applied to an asymmetric NACA 0015 hydrofoil (Fig.4), with a mean camber line given by $y_{c}=4 x-4 x^{2}$. The ratio of sagitta to chord length is 0.1 . Figures 5 and 6 show the results of this case. The obtained results show that:

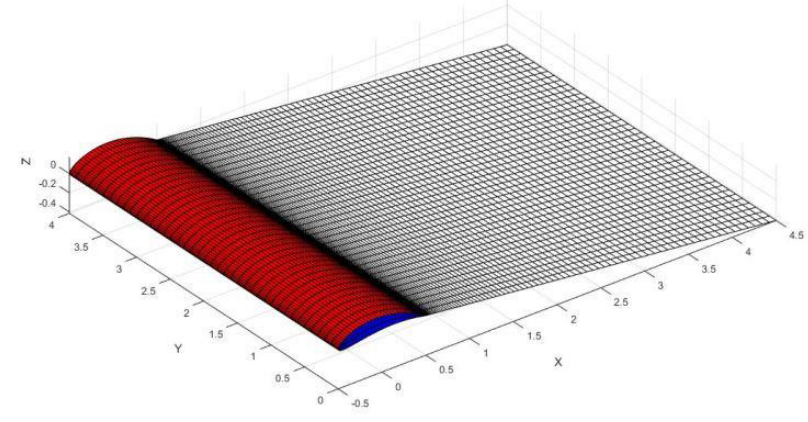

Figure 4. Discretization of asymmetric NACA-0015 hydrofoil

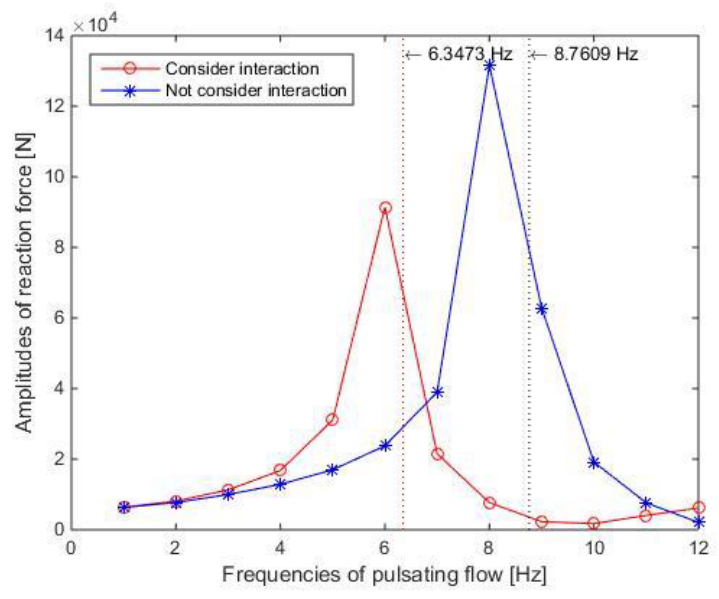

Figure 5. Reaction amplitudes for vary ing pulsating flow frequency for the asymmetric NACA0015 hydrofoil.

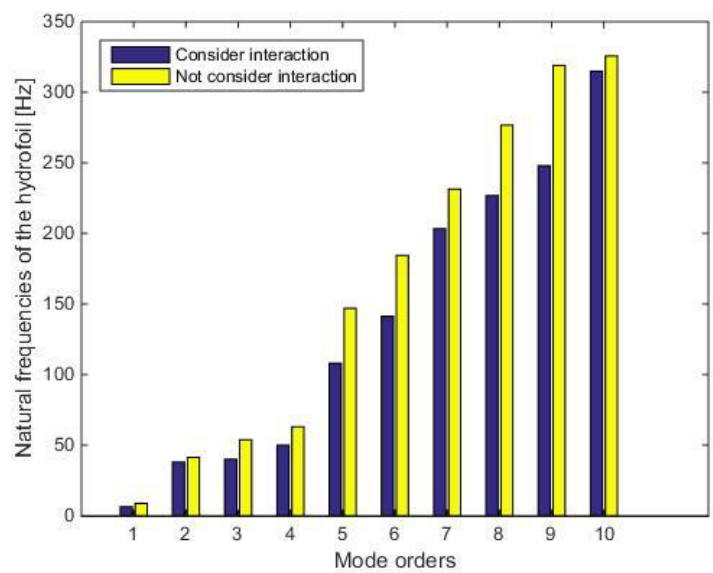

Figure 6. Natural frequency comparison for the asymmetric hy drofoil.

1. No contradiction has been found with the classical vibration theory.

2. The natural frequencies decrease when the fluidstructure coupling matrices are considered.

3. The amplitude of the support reaction at the first natural frequency is less when the fluid-structure coupling matrices are considered.

\section{Conclusions}

This paper has presented a theoretical and numerical method to study fluid-structure interaction of hydrofoils in a pulsating flow. Both symmetric and asymmetric hydrofoils have been investigated by the combined codes. Results reveal that: 1) natural frequencies of hydrofoils have a significant reduction when the effects of fluidstructure coupling are considered. 2) the amplitude of the support reaction at the 1 st natural frequency is approximately 33\% less when the fluid-structure coupling matrices are considered. 3) fluid-structure interaction plays an important role in the performance of elastic hydrofoils, even though the elastic deformations are small.

\section{References}

1. Jiho You, Jinmo Lee, Donghyun You, Optimization of propulsion kinematics of a flexible foil using integrated CFD-CSD simulations, International Mechanical Engineering Congress \& Exposition, (2012)

2. Suzanne Ruth Hutchison, Numerical Modelling of Hydrofoil Fluid-Structure Interaction. Doctoral diss ertation (2012)

3. Y.L. Young. Time-dependent hydro-elastic analysis of cavitating propulsors. Journal of Fluids and Structures, 23 :269-295 (2007)

4. Y.L.Young. Fluid - structure interaction analys is of flexible composite marine. Journal of Fluids and Structures, 24 :799-818 (2008) 
5. Huei-Jeng Lin, Jiun-Jyi Lin, Nonlinear hydroelastic behavior of propellers using a finite element method and lifting surface theory. Mar Sci Technol, $1: 114-$ 124 (1996)

6. Liu Zhen, Hyun Beom-soo, Kim Moo-rong, Jin Jiyuan. Experimental and numerical study for hydrodynamic characteristics of an oscillating hydrofoil. Journal of Hydrodynamics, Ser. B, Volume 20, Issue 3, Pages 280-287 (2008)

7. O. De La Torre,X. Escaler, E. Egusquiza,M. Farhat. Numerical and experimental study of a nearby solid boundary and partial submergence effects on hydrofoil added mass. Computers \& Fluids 91 (2014)

8. Hess, J. L. and Smith, A. M. O. Calculation of nonlifting potential flow about arbitrary threedimensional bodies, The Journal of Ship Research,No. 8, pp. 22-44 (1964)

9. Hess, J. L. and Smith, A. M. O. Calculation of potential flow about arbitrary bodies, Progress in Aeronautical Sciences, Vol. 8, pp. 1-138 (1967)
10. Kuo, J. Analys is of propeller blade dynamic stresses. Doctoral dissertation, Department of Naval Architecture and Marine Engineering, The University of Michigan, Ann Arbor, MI. (1984)

11. Mohammad Shahjada Tarafder*, Goutam Kumar Saha*, and Sayeed Tanvir Mehedi. Analysis of Potential Flow around 3-dimensional Hydrofoils by Combined Source and Dipole Based Panel Method. Journal of Marine Science and Technology, Vol.18, No.3, pp.376-384 (2010)

12. Hassan Ghassemi, Ahmad Reza Kohansal, Numerical evaluation of various levels of singular integrals, arising in BEM and its application in hydrofoil analysis, Applied Mathematics and Computation, 213: 277-289 (2009)

13. Xiao-Wei Gao, Evaluation of regular and singular domain integrals with boundary-only discretization - theory and Fortran code. Journal of Computational and Applied Mathematics, 175 : 265$290(2005)$ 